\title{
ESTUDIO LÉXICO DE VERBOS DE TRANSFERENCIA + CLÍTICOS EN LA VARIEDAD ARGENTINA DEL ESPAÑOL
}

\section{LEXICALIST ACCOUNT OF VERBS OF TRANSFER + CLITICS IN THE ARGENTINIAN VARIETY OF SPANISH}

\author{
Cadina Mariel Palachi \\ Universidad Nacional del Litoral \\ cadina.palachi@gmail.com
}

\section{RESUMEN}

Este trabajo analiza, en el marco de las teorías generativistas sobre el léxico, verbos cuyo significado de transferencia cambia en presencia del clítico las en la variedad argentina del español, como en (1). Se trata de predicados complejos o locuciones idiomáticas que, a diferencia de las construcciones tratadas en la bibliografía (generalmente verbo + nombre $-\mathrm{o}$ sintagmas nominales/determinante) y cuyo peso semántico se suele atribuir a la unión de ambos constituyentes, están conformadas por un verbo + clítico(s). En los ejemplos de (1), el verbo y el clítico forman una unidad de sentido sin que a ninguno de los dos pueda atribuírsele una aproximación al significado resultante de la unión, puesto que el verbo modifica su significado estándar. Fundamentalmente, el clítico (las) parece no tener referencia posible y se comporta casi como un expletivo.

(1)

a. Se las tomó (se fue) / Se la tomó en serio.

b. Se las da de mucho / Juan se las da. (es soberbio)

c. Juan se mandó nomás.

d. Juan se la manda siempre.

e. Se la tira de inteligente / de vivo.

f. Jimena Barón la rompió en el Bailando.

¿Cuál es el procedimiento de creación léxica que produce este tipo de modificaciones en el significado verbal? ¿Es un mecanismo de modificación de los rasgos semánticos o es de tipo morfológico? En cualquier caso, cabe preguntarse también cuáles son los rasgos fundamentales del verbo que permanecen en el resultado y cuáles cambian.

Este trabajo plantea preguntas para futuras investigaciones especialmente relacionadas con el estatuto categorial del clítico y la posibilidad de realizar un estudio comparativo con otras variedades del español.

Palabras clave: léxico, español, variedad argentina, verbos, clíticos referencia

\section{Abstract}

This paper analyzes, within the framework of generative grammar, verbs of transfer that, in the argentinian variety, modify their meanings with the clitic las, as in (1). Complex predicates or idioms, but unlike the constructions usually analized in the literature (verb + noun) whose semantic weight is usually attributed to the union of both constituents, these are conformed by a verb + clitic(s). In the examples (1) the verb and the clitic form a unit of sense but neither of them can give an approximation to the meaning of the resulting form. The verb modifies its basic meaning and the clitic (las) seems to have no possible reference and behaves like an expletive.

(1)

a. Se las tomó (se fue) / Se la tomó en serio.

Herself them take-past/ herself them take-past seriously

She leaved / She made things seriously.

b. Se las da de inteligente/ Juan se las da.

Himself them gives of intelligent / John himself them give

He is arrogant.

c. Juan se mandó nomás.

John himself order-past nomás

John did something (without analyzing the consequences of his acts).

d. Juan se la manda siempre.

John himself it order-pres. always

John always do things wrong.

e. Se la tira de inteligente.

Himself them throw of intelligent

He makes the other see that he is intelligent / clever

f. Jimena Barón la rompió en el Bailando.

Jimena Barón it brake-past in the Bailando.

Jimena Barón did it the best in the Bailando.

Which is the procedure of lexical creation that changes the verbal meaning? Is it a semantic mechanism or is it a morphological one? What are the fundamental features of the verb that remain in the result and which change?

This work raises other questions for future research related to the categorical status of the clitic and for comparative study with other varieties of Spanish.

Keywords: lexic, Argentinian variety of Spanish, verbs, clitics, reference 


\section{EL PROBLEMA}

Las expresiones de (1) configuran diferentes tipos de predicados complejos o locuciones idiomáticas, pero, a diferencia de las construcciones de este tipo tratadas en la bibliografía (Mendivil Giró, 1999), estas están conformadas por un verbo + clítico(s). En la formación de elementos léxicos, un procedimiento bastante extendido en las lenguas consiste en conformar locuciones verbales a partir de un $\mathrm{V}$ y su SN-Complemento (hacer mención, meter la pata) cuyo resultado son sintagmas con significados que van desde ser relativamente predictibles a totalmente impredecibles a partir de la suma de sus partes, y que adquieren el estatuto de ítems léxicos. En ese tipo de compuestos sintagmáticos el peso semántico, aunque no el categorial, puesto que el resultado es una locución con categoría verbal, suele recaer en el SN (SD) como elemento significativo central.

En cambio, en los ejemplos de (1) (y también en los de 2 y 3, pero no nos ocuparemos de ellos por las razones que se mencionan más abajo) el verbo y el (o los) clítico/s conforman una unidad de sentido sin que a ninguno de los dos pueda atribuírsele una aproximación al significado resultante de la unión, es interesante notar que una traducción al inglés (o a cualquier otra lengua) genera ciertos inconvenientes para encontrar la expresión adecuada similar. En el mismo sentido se hace necesario glosar su significado para que se comprenda en la variedad estándar. El clítico parece no tener referencia posible y se comporta casi como un expletivo.

a. Se las tomó (se fue) / Se la tomó en serio.

b. Se la da de mucho / Juan se las da. (es soberbio)

c. Juan se mandó nomás. (hizo algo sin dudarlo mucho)

d. Juan se la manda siempre (hace las cosas mal)

e. Se la tira de inteligente / de vivo. (cree que es inteligente)

f. Jimena Barón la rompió en el Bailando. (Hizo un papel brillante)

a. Se da con todo / marihuana / cocaína / vodka. (se droga, se alcoholiza)

b. Le dio / le da (lo somete a un acto sexual)

c. "En los recitales nuestros se canta que Cerati se la come y que el Indio se la da, y en los de ellos al revés." (Indio Solari, 1997, Clarín).

(3)

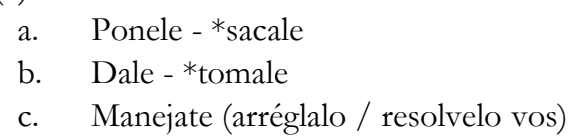


Los ejemplos de 1 se diferencian de los de 2 y de los de 3 en varios sentidos:

En (1) el clítico (las, la) no tiene referencia de ningún tipo (se las da), no se puede reemplazar por ningún SN. En (2) (le da, se da, se la come) se puede pensar un SN, aunque sea con un sentido figurado o metafórico, tal como se ve en le da a Cerati, la come (al órgano sexual masculino, generalmente designado con sustantivos en femenino) al que hace referencia el clítico. En (3) inferimos que hay una gramaticalización ${ }^{1}$ de los verbos de transferencia (dar/poner/¿manejar?), que se hace más evidente si comparamos con la imposibilidad de los verbos de transferencia inversos: sacale, tomale y recibile que, al no encontrarse gramaticalizados como sus contrarios, solo podrían emplearse con sus significados de diccionario. También es posible argumentar que se ha producido un proceso de gramaticalización si se tiene en cuenta la imposibilidad de estos verbos para flexionarse en los usos de (3): *démosle, *denle, *pongámosle.

En los ejemplos de (1) los verbos cambian sus significados léxicos. En (2) tienen un significado ligeramente diferente pero no pierden totalmente su rasgo de transferencia.

Los verbos dar, tomar y mandar son verbos que en su sentido literal expresan la transferencia y tienen tres argumentos (agente, tema, locativo), tirar y romper, no presentan el rasgo de transferencia, pero creemos que pueden entenderse como verbos de movimiento y de cambio de estado, respectivamente y rigen dos argumentos (agente, tema).

\section{EL PUNTO DE PARTIDA. OTROS ESTUDIOS SOBRE EL TEMA}

Si bien el fenómeno que nos ocupa aquí ha sido notado por muchos autores que se ocupan de la gramática del español, especialmente por aquellos que se han dedicado a estudiar las características de las variedades hispanoamericanas ${ }^{2}$, hay dos trabajos que son particularmente relevantes pues plantean aproximadamente el mismo tema que

\footnotetext{
${ }^{1}$ Entendemos la gramaticalización como el proceso lingüístico por el que un ítem léxico adquiere valor funcional. Haría falta analizar más en profundidad estos casos. Aquí mencionamos que creemos que en el caso de "dale", "ponele" y "manejate", la gramaticalización se encuentra en un grado medio para los dos últimos. En cambio, "dale” ha adquirido un valor de partícula discursiva.

${ }^{2}$ El trabajo de Ghio y Albano (2014) presenta un recorrido interesante de bibliografía sobre el tema.
} 
aquí nos ocupa. Se trata del trabajo de Delbecque (2009) quien centra su interés en el comportamiento del pronombre femenino plural sin referencia. Realiza un análisis exhaustivo del comportamiento del pronombre y considera a estas unidades como lexicalizaciones, teniendo en cuenta que el significado no se puede obtener a partir de la suma de sus partes.

Ghio y Albano (2015) realizan un exhaustivo inventario y clasificación de los tipos de construcciones en los que se encuentra un clítico las, cuya referencia no está totalmente determinada. Estos autores no consideran a los compuestos resultantes como lexicalizaciones. Clasifican las construcciones posibles en cinco tipos: verbos transitivos que no modifican sus significados, verbos transitivos más clítico y predicativo objetivo, verbo transitivo más clítico y objeto indirecto, verbo intransitivo más clítico la y verbos pronominales transitivos o intransitivos.

Justamente en este último grupo entra el tipo de verbos que nos interesan y es allí donde los autores indican la complejidad que revisten estos verbos y la dificultad para sistematizarlos. Expresan con claridad que el cambio de significado del verbo puede tener un origen diacrónico relacionado con la evolución de un sintagma nominal o de determinante que se usaba habitualmente en el contexto de estos verbos.

Nuestro trabajo toma como punto de partida la clasificación realizada pero se detiene en el significado de los verbos en ausencia y en presencia del clítico. Especialmente, teniendo en cuenta que los mismos verbos pueden coaparecer con un clítico acusativo, femenino, plural con referencia y, en tal caso, tienen un significado registrado en los diccionarios.

\section{LAS PREGUNTAS}

Las preguntas que surgen a partir de estos ejemplos se pueden clasificar según se refieran a la solución que se da en los diccionarios y a la descripción de las características sintácticas, morfológicas y semánticas de los elementos analizados.

Nos interesa observar cómo se registran estas locuciones en los diccionarios. Dado que los nuevos significados de los verbos no parecen poder deducirse de la unión de $\mathrm{v}+\mathrm{cl}$ ¿El Diccionario de Habla de los Argentinos (2008) de la Academia Argentina debería 
registrarlos? ¿Las registran los diccionarios generales más importantes de la lengua española?

¿Qué características definen a estas locuciones? ¿Se encuentran totalmente o semilexicalizadas en la variedad argentina? ¿Cuál es el procedimiento de creación léxica que produce este tipo de modificaciones en el significado verbal? ¿Es un mecanismo morfológico?

A partir de estas preguntas surgen otras ¿Qué tipo de elemento es el clítico en estos ejemplos? ¿Un N vacío (Panagiotidis 2002, p. 85) o expletivo? ¿Se trata de un V liviano $+\mathrm{N}$ o el clítico se comporta como un afijo derivativo? ¿Es posible formar locuciones de este tipo en otras variedades del español?

\section{LOS DICCIONARIOS}

En las entradas correspondientes a tomar y dar del Diccionario de Habla de los Argentinos (2008) no se registran estos sentidos. Cuando se trata de dar liviano $+\mathrm{N}$, el diccionario presenta algunos significados en la entrada del $\mathrm{N}$ correspondiente: dar asco, pero el clítico no aparece en el diccionario porque es un elemento funcional. ¿Cómo se podría inferir el significado de dárselas? Con respecto a tomárselas, el Diccionario de Uso del Español de América y España (2012) de VOX (VOXUSO), registra:

(4) Tomárselas: Río de la Plata, Bolivia, Coloqu. Irse [una persona] de un lugar por conveniencia, a veces evadiendo una responsabilidad o para evitar un compromiso.

Ej. Se las tomó antes de que su jefe le pidiera más cosas y tuviera que quedarse hasta las mil y quinientas. (VOXUSO, 2012 [formato CD]: s.v. tomar).

El Diccionario de la Real Academia Española on line (DLE, obtenido de: http:/ / dle.rae.es, 2014) no lo registra.

Un ejemplo interesante es el diccionario de insultos de la Revista Barcelona, Puto el que lee (2006, p. 63), que presenta el siguiente significado para un verbo que no analizamos aquí; poner. Este verbo pierde sus dos argumentos internos (tema, locativo) y adquiere significado de verbo pleno, aunque requiere la presencia del dativo, por un mecanismo mucho más radical que el que analizamos aquí, es decir, no se retiene el clítico vacío para modificar el significado del verbo, poner, con el significado de dar una piña / pegar. 
Sino que se sobreentiende el complemento directo (una piña) y también en otros contextos adquiere un significado de 'insulto por invitación'.

(5) Poner: Pegar, dar una piña. "si no parás de insultarme, te voy a poner y te voy a dejar la cara morada." // Para Barthes, el verbo también se utiliza en insultos de invitación "Ponete en cuatro que te parto el orto en cuatro".

En lo que se refiere al verbo mandar, el VOXUSO (2012) da cuenta de un mecanismo similar de formación, en el español coloquial de México, pero con un resultado diferente en cuanto al significado final:

(6) verbo pronominal

8. mandarse. Méx coloquial. Excederse con un dicho o un hecho con relación a alguien o algo: ej no te mandes con los gastos. (VOXUSO, 2012: s.v. mandar).

En (6) se puede observar que este procedimiento es bastante similar al que nos ocupa aquí, pero no totalmente, puesto que si bien el $\mathrm{V}$ adquiere un significado novedoso en presencia del clítico se y se torna un verbo pronominal, no se encuentra la presencia de un clítico en acusativo y sin referencia. De todas maneras, creemos que, a partir de esta entrada, es posible hipotetizar que este tipo de formación de nuevos significados se podría encontrar en otras variedades de la lengua. En este mismo sentido encontramos en el DLE (2014, online) verbos como liarla y su variante más actual liarla parda, en los que, si bien no se registra la presencia del clítico reflexivo se, el pronombre en acusativo femenino singular (la) parece no tener una referencia clara. Haría falta un análisis mucho más detallado de estos casos, pero creemos que el mecanismo de formación que nos interesa tiene realizaciones posibles en diferentes variedades del español.

\section{RECURSOS DE LA CREACIÓN LÉXICA}

Nos interesa considerar aquí el tipo de procedimiento por el que es posible que elementos léxicos ya existentes en una lengua adquieran nuevos valores. Cabré (2008, p. 14) afirma que la neología es el "fenomen consistent a introduir en una llengua una unitat lèxica o un altre recurs lingüístic nous, que o poden haver estat creats aprofitant els recursos interns de la pròpia llengua o bé manllevats a una llengua forana”. También señala Cabré (2008) que, una vez que el neologismo ingresa en el diccionario, deja de 
serlo. En el caso de las locuciones que analizamos aquí, nos parece interesante destacar que se trata de creaciones léxicas de una comunidad que difícilmente puedan ingresar en otras comunidades y en este sentido entendemos que estas locuciones, que en su momento fueron neologismos, han sido introducidas en el diccionario como propias de una variedad. Pero también entendemos que, más allá del ingreso de estas locuciones en el VOXUSO (2012), el mecanismo de creación debe ser explicado. Según la clasificación de Guilbert (citado por Cabré, 2008), los neologismos pueden ser denominativos o estilísticos. Los primeros se crean para dar nombre a nuevos conceptos y los segundos, en cambio, son el producto de las necesidades expresivas de un grupo o una comunidad.

Entendemos que los elementos léxicos que aquí consideramos han sido neologismos estilísticos. Es necesario señalar que hay expresiones propias de la lengua estándar que tienen un significado proposicional idéntico, pero son diferentes estilísticamente y existen mayores posibilidades de que sean producidas en contextos y registros distintos, como se observa en (6) y (7):

(6) Me voy a mi casa porque ya es tarde.

(7) Me las tomo porque ya es tarde.

Por otra parte, creemos interesante señalar que se trata de unidades formadas por el cambio de significado de otras ya existentes en la lengua. Por lo que entendemos que se trata de 'neologismos semánticos'.

Interesa, entonces, revisar cuáles son los procedimientos de formación de conceptos que se ponen en juego. Analizaremos por separado ambos elementos; el clítico y la semántica de los verbos para luego ponerlos en relación.

\section{LOS CLÍTICOS}

Las formas tomárselas, mandársela, dárselas deben ser descriptas considerando los tipos de clíticos que las conforman.

El clítico se es una forma del pronombre variable puesto que admite el cambio de persona y número: 
(8) Juan, tomátelas.

(9) Chicos, Uds., dejen de mandársela que ya tenemos suficientes problemas.

(10) Bueno, podemos dárnoslas de inteligentes pero sabemos que no es así.

Se trata de una pronominalización del verbo que es muy común en el español.

En cambio, el clítico la/las se nos presenta como más problemático porque no solo no es variable en cuanto a la flexión de persona (*tomátenos, *dársete), sino que tampoco admite variación de caso, ni de género (*digo que se les tome yal \#digo que se los tome ya). En cuanto al número, entendemos que admitiría cierta flexibilidad pero creemos que, si bien haría falta un análisis más profundo, tal vez esta variación posible solo se deba a una cuestión fonológica, ligada a la capacidad de caer que tiene el fonema / s / en final de palabra, especialmente en la variedad santafesina del español ${ }^{3}$. Las dos formas de (11) son posibles:

(11) Juan, tomátela / Juan tomátelas.

¿Los clíticos son siempre constituyentes sintagmáticos argumentales? Muchos lingüistas ${ }^{4}$ coinciden en caracterizar a los clíticos como afijos frasales o sintagmáticos, porque, en palabras de Camacho Taboada (1998, p. 82) "si bien, fonológicamente, se adjuntan a un anfitrión linealmente próximo, desde el punto de vista de la estructura constitutiva, lo hacen a nivel frasal o clausal". Sin embargo, la autora indica a continuación la dificultad de considerar esta definición como apropiada, especialmente en aquellas lenguas en las que el clítico termina formando parte de la palabra como un afijo flexivo (porque hace concordancia con el verbo), y de esta manera se transforma en un elemento no frasal. Para ser un elemento frasal hace falta que constituya un sintagma (un $\mathrm{N}$ proyecta en $\mathrm{SN}$ ) y que desempeñe una función (complemento, adjunto).

\footnotetext{
${ }^{3}$ Cabe aclarar aquí que nuestro trabajo toma como objeto de estudio específicamente el español de Santa Fe y Paraná, dentro de la variedad argentina. Es importante destacar que las expresiones que analizamos están extendidas por el territorio del país, pero los rasgos fonéticos, no. En Santa Fe, especialmente, por la influencia de la inmigración italiana está bastante extendida la pérdida del fonema /s/ en final de palabra, sin que por este motivo se pierda la flexión, por ejemplo, de plural, en ocasiones con una leve aspiración final o sin ella.

${ }^{4}$ Camacho Taboada (1998) que cita los trabajos de Zwicky (1977, 1985), Klavans (1982, 1985), Kaisse (1985), Nevis (1985) y Anderson (1993, 1996)
} 
Los clíticos que nos ocupan parecen ser expletivos (como it, it) puesto que no pueden ser llenados referencialmente. Pero a diferencia de estos, tienen efectos léxicos y semánticos sobre el significado del verbo al que se unen. Sin embargo, también pueden interpretarse como elementos argumentales. El clítico las ha perdido su capacidad para aparecer en una función sintáctica por sí mismo pero conserva el caso acusativo.

\section{DESCRIPCIÓN DE LA ESTRUCTURA ARGUMENTAL Y DEL SIGNIFICADO DE LOS VERBOS}

La característica que comparten los verbos tomar, dar y mandar (algo a alguien) en sus significados estándares, es el rasgo semántico de transferencia de posesión y, por lo tanto, de movimiento.

Para realizar un análisis adecuado de la semántica léxica de estos verbos recurriremos a un marco teórico que, según entendemos, es muy potente y tiene una gran capacidad descriptiva. La Estructura Léxico Conceptual de un ítem, según Jackendoff (1990), es una entidad formada por constituyentes conceptuales que pertenecen a un pequeño conjunto de categorías ontológicas mayores: cosa, evento, estado, acción, lugar, trayecto, propiedad y cantidad. Combinados, estos constituyentes, o rasgos, permiten la creación de infinitos ítems léxicos. Algunos de estos constituyentes establecen relaciones, de manera que requieren elementos que llenen los espacios argumentales que dejan abiertos, es decir, son variables en la estructura léxica que deben ser ligadas en la sintaxis.

Una idea importante de esta teoría es que las formalizaciones para codificar los conceptos de locación espacial y movimiento, abstraídos correctamente, pueden ser generalizados a muchos otros campos semánticos. De allí que la "transferencia” pueda asociarse con el "movimiento", puesto que las entidades transferidas son, de hecho, puestas en movimiento.

La ELC que proponemos para el verbo tomar en su acepción estándar es la siguiente:

(12) [Evento CAUSAR ([cosa $\left.\mathrm{X}_{\mathrm{i}}\right]$ ) [Evento IR [Posesión], [Trayecto DESDE [lugart], [trayecto HACIA $\left.\left.\operatorname{COSA}_{\mathrm{i}}\right]\right]$ 
Para el verbo dar proponemos la ELC inversa:

(13) [Evento CAUSAR ([Cosa $\left.\mathrm{X}_{\mathrm{i}}\right]$ ) [Evento IR ([Posesión], [trayecto DESDE COSA $\left.\left.\mathrm{i}\right]\right)$, [trayecto HACIA ([lugart)]

Considerar verbos como tomar y dar como eventos de movimiento permite pensar su significado en tanto constituido por rasgos sometidos a los principios de la formación semántica y subsumirlos dentro de la clase más general de los predicados que expresan el movimiento. Por otra parte, estas EELLCC dan cuenta de la estructura argumental de los predicados que estamos considerando. Los tres espacios que quedan "abiertos", deberán ocuparse en la sintaxis por medio de argumentos. También hay que observar que la función COSA de CAUSAR se encuentra coindizada con la función COSA de TRAYECTO HACIA / DESDE. Por lo tanto, se entiende que el causante es también el punto de llegada / origen de la transferencia, es decir del movimiento.

En (15) presentamos un ejemplo en el que la estructura del verbo DAR de transferencia de posesión presenta los argumentos requeridos y en (16) el uso coloquial del español de la variedad argentina en el que el clítico las no tiene referencia y el verbo cambia su significado:

(14) Juan tiene unas carpetas para Miguel. No sabe si dárselas ahora o más tarde.

(15) Ese Juan, que deje de dárselas (de inteligente) porque ya molesta.

(16) [Evento CAUSAR ([Cosa Juan $\mathrm{i}$ ] ) [Evento IR ([Posesión Cosa las], [trayecto DESDE (COSA $\mathrm{i}]$ ), [trayecto HACIA ([lugar le/a Miguel])]

En esta ELC se puede ver que el argumento más cercano al V se realiza mediante un clítico en acusativo (las) que tiene una referencia (en este caso "las carpetas") y el clítico le (en este caso en su forma se por una cuestión fonética) reduplicado como "a Miguel", en dativo es el "destino".

Esta misma ELC no es posible para el ejemplo de (16), puesto que el clítico se no ocupa la misma posición argumental que el del ejemplo en que DAR es un verbo de transferencia. Sumado a esto, el clítico las no parece poder ir insertado como argumento Acc del verbo. Creemos que el cambio de significado de los verbos se produce por la modificación de rasgos semánticos asociados al cambio de estructura argumental. 
La propuesta para el nuevo significado de este verbo es que los clíticos han dejado de ser argumentales y se han integrado al verbo [v dárselas], un tipo de elemento léxico que puede ir acompañado de un complemento regido, encabezado por la preposición de. Ejemplos como dárselas de pobrecito o dárselas de vivo, lo demuestran.

Consideremos ahora la estructura del verbo tomar que, en su significado estándar, puede referirse a varios tipos de eventos. Una de las acepciones básicas se presenta como el evento de hacer que un objeto pase a estar en posesión de alguien (especialmente para sí mismo). En otras acepciones puede también entenderse como en (18) con el sentido de beber. Los ejemplos de (19) y (20) tienen diferentes significados:

(17) Había unas carpetas sobre la mesa de Pedro. Juan las tomó sin preguntar.

(18) Le regalaron unas cervezas a Juan. Se las tomó (todas) en seguida. / Las tomó ese mismo día.

(19) Juan era un pesado. Así que María se las tomó en seguida.

Se puede observar, por el contexto oracional inmediato, que en (18) y también en (19) hay un sintagma nominal (o SD) cuya referencia es retomada luego por el clítico las; en (16) unas carpetas, en (19) unas cervezas. Por su parte, en (20) no hay posibilidad de recuperar la referencia del clítico.

La ELC de (21) representa la interpretación de tomar en el uso de (18):

(20) [Evento CAUSAR ([Cosa Juan]) [Evento IR [Posesión unas carpetas], [Trayecto DESDE [lugar la mesa]], [trayecto HACIA Juan $i$ ]]]

Hay que hacer notar que en la variedad argentina, especialmente rioplatense, la acepción de tomar como lo define en su primera y segunda entradas el DLE (2014, online): "coger o asir con la mano algo, coger aunque no sea con la mano”, es quizás la menos habitual. La otra posibilidad, con el significado de beber, se encuentra registrada en el DLE (2014, online) en la entrada 6. Sin embargo, la entrada correspondiente indica: "comer o beber". Se debe mencionar que en la variedad que estudiamos, el sentido de tomar equivalente a comer no es posible.

Es importante señalar las diferencias en cuanto al pronombre se en los ejemplos de (19) y (20); cuando el verbo tomar tiene el significado de beber puede llevar o no el pronombre se que, cuando está presente, tiene un valor aspectual de culminación. Así en el ejemplo de (19) se utiliza especialmente en presencia del sintagma de 
cuantificador todas, aunque la ausencia del mismo no cambia la lectura cuantificada. En el ejemplo de (20) el pronombre se presenta un valor también aspectual, pero de incoatividad, de manera que, entre los muchos posibles valores semánticos del verbo tomar, el más básico, la primera entrada de DLE (2014, online), no es pronominal pero rige un SD complemento o bien un pronombre variable en caso acusativo: lo/los-la/las que ocupa una posición argumental de Complemento Directo. En el sentido del verbo como beber, un uso muy común en la variedad argentina, el pronombre en acusativo es un argumento del verbo y tiene la función sintáctica de Complemento Directo (también es variable en género y número y está en caso acusativo). Por su parte, el clítico las, correspondiente a la expresión que nos ocupa; tomárselas, no es variable, no está en una posición argumental y no posee una referencia posible, de manera que no podría ser intercambiado por un SN/SD en acusativo.

Es cierto que el clítico las puede haber tenido una referencia en algún momento diacrónicamente no muy lejano, ya que varias personas nos han señalado que originalmente se podía decir tomar las de Villadiego ${ }^{5}$, aunque esas mismas personas reconocen no saber que esta localidad está situada al norte de España, incluida una informante española. De manera que mi hipótesis sobre la pérdida del rasgo referencial en esta expresión podría ampliarse hasta llegar a postular que el SD "las de Villadiego" ya había sufrido un proceso de lexicalización, en la medida en que se había integrado al verbo pronominal tomarse para dar lugar a un compuesto con significado diferente al del verbo tomar en sí mismo.

Borer (1986) señala que los pronombres clíticos típicamente satisfacen los requerimientos de subcategorización de los verbos y, en este sentido, se entiende que tiene la misma función que el SN correspondiente. Esta conclusión tiene algunos problemas, como hace notar la misma autora, porque los clíticos que entran en la formación de palabras no tienen la estructura interna propia de un $\mathrm{SN}$, no tienen especificadores, ni modificadores, ni complementos. De manera que se puede concluir, también siguiendo a Borer (1986), que los clíticos son afijos. Creemos que la

\footnotetext{
${ }^{5}$ Este mismo dato lo mencionan Ghio y Albano (2015) a partir de una consulta al diccionario de María Moliner.
} 
conclusión de Borer (1986) permitiría explicar la formación de las locuciones que presentamos aquí, pero persistiría el problema de explicar los casos, mucho más habituales, en los que el clítico ocupa una posición argumental.

Una de las hipótesis más fuertes de la teoría lexicalista generativa es que la ELC de un ítem determina su sintaxis, esta hipótesis se enmarca en el proyeccionismo. Si en una única variedad conviven varias acepciones de un mismo ítem léxico, la pregunta que habría que hacerse sería: ¿cómo es posible que el hablante pueda determinar inmediatamente el significado adecuado en cada caso? Y si se trata de una misma pieza léxica con muchos significados que determinan su sintaxis, ¿cómo se encontraría almacenada en el cerebro de los hablantes?

Las teorías sintactistas de la formación del léxico presentan hipótesis más adecuadas para responder a estas cuestiones. Nuestros datos parecen avalar un análisis basado en la Morfología Distribuida, de acuerdo con la cual la formación de los elementos del léxico se produce en la sintaxis, y según sus principios. El significado es una consecuencia de la estructura sintáctica y del tipo de morfemas, así como de argumentos que entran en relación de acuerdo con los principios de la sintaxis y no a la inversa. Creemos que, tal como han señalado los mismos teóricos lexicalistas, la posibilidad de entender que la ELC de un ítem responde a los principios de la sintaxis, permite pensar que la formación que hemos propuesto, siguiendo a Jackendoff (1990), es correcta. La diferencia estaría entonces en que los ítems, al formarse en la sintaxis derivarían su significado de la estructura que presentan y no a la inversa.

\section{GRADO DE LEXICALIZACIÓN}

El concepto mismo de lexicalización, como señala Buenafuentes de la Mata (2007), siguiendo en esto a varios autores, es controvertido y está menos claro que el de gramaticalización. Sin embargo, interesa la definición que propone Buenafuentes de la Mata (2007, p. 35) “en un sentido estricto", quien afirma que la lexicalización transforma un elemento gramatical en uno léxico. El proceso por el cual un clítico (que 
ha perdido su rasgo referencial) se integra a una palabra dando lugar a un significado nuevo, en nuestra opinión, puede ser entendido como un proceso de lexicalización. ${ }^{6}$

Creemos que las locuciones verbales que estamos analizando se encuentran en un proceso de lexicalización que no ha alcanzado su grado máximo.

El proceso de lexicalización alcanzado por una locución varía desde un grado bajo en el que es posible incluir elementos entre los constituyentes que la forman o modificar el orden de los mismos, así como flexionarlos en todas las formas posibles del paradigma hasta un grado en el que ninguna de estas operaciones es posible.

Teniendo en cuenta lo mencionado, es pertinente realizar algunas pruebas sintácticas que nos permitan observar en qué grado se encuentran lexicalizados estos ítems.

Las estructuras en las que los clíticos tienen un verdadero valor argumental pueden servir de contracara a las locuciones en las que los clíticos están fosilizados con el verbo. Por este motivo nos detendremos en una comparación entre la estructura del verbo con su significado básico de diccionario y las expresiones que analizamos en este trabajo.

En primer lugar, respecto de la flexión, ya hemos notado que el clítico las de estas locuciones no puede presentarse en otro género o número, solo en femenino plural. En cuanto al clítico se, si bien cuando el verbo lleva su significado de diccionario admite variación de persona y número, cuando se trata de la locución que nos interesa, está mucho más restringido:

(21) a. Nos tomamos unas cuantas cervezas en la casa de María.

b. Me tomé una cerveza en la casa de María.

c. Nos creímos todo lo que nos dijo.

(22) a. Juan se las tomó rápido anoche.

b. Me las tomé rápido ayer para no ver a Juan.

c. ?Me las doy porque soy inteligente.

d. ??Nos las tomamos rápido ayer para no ver a Juan.

e. ?Se las da porque es inteligente.

f. ??Nos las damos porque somos inteligentes.

${ }^{6}$ La discusión acerca de los conceptos de lexicalización y gramaticalización es interesante, sin embargo, no la desarrollaremos aquí porque no constituye el objeto central de nuestro trabajo. 
En cuanto al verbo, también es posible examinar su capacidad para variar de persona, número, tiempo y modo.

(23) a. Tomátelas Juan.

b. ?? ¿A qué hora te las tomarás?

c. ¿A qué hora te las vas a tomar?

d. ¿Ya se las habrá tomado Juan?

e. Espero que Juan ya se las haya tomado cuando lleguemos.

f. ? Es posible que Juan se las tomase más temprano, porque no lo vimos.

Se puede observar que la variación del verbo en cuanto a tiempos y modos es bastante admisible, en cambio, es más resistente a la modificación de persona. Inferimos que esto se debe a la necesidad de modificar al mismo tiempo la persona del clítico se.

Una prueba que da buen resultado con los mismos verbos en contextos donde los clíticos son argumentales, pero no así cuando se trata de elementos lexicalizados, es la elevación del clítico.

(24) Juan le da muchas flores a María. Que se las deje de dar porque no le gustan.

(25) Juan se las da todo el tiempo. *Que se las deje dar porque molesta.

(26) Juan quiere tomar una cerveza cuando sale. ¿La puede tomar aquí?

(27) Juan tomátelas ya. ¿Te las podés tomar ya, Juan? / ¿Podés tomártelas ya Juan?

Se observa que la estructura de elevación del clítico de (26) da cuenta de una lexicalización bastante avanzada, sin embargo, en (27) la locución verbal tomárselas admite la elevación de clítico con el auxiliar poder.

Tampoco la pasivización es posible, como consecuencia casi inevitable de la falta de referencia del clítico:

(28) Juan le dio estas revistas a María. Estas revistas fueron dadas a María (por Juan).

(29) Juan se las da. \#Estas fueron dadas por Juan.

Ni la interrogativa de complemento directo, ni indirecto:

(30) ¿Qué le dio Juan a María? ¿A quién le dio Juan las revistas?

(31) \#¿Qué se da Juan? \#ंA quién las da Juan?

(32) \#¿Qué se tomó Juan?

El grado de lexicalización de estas locuciones es bastante elevado. Si tomamos en consideración que el significado de los constituyentes aislados no permitiría predecir el significado de la expresión y la incapacidad de los clíticos para presentar variación 
morfológica, estamos en condiciones de afirmar que estas locuciones podrían entrar en el diccionario con sus significados impredecibles a partir de los de sus constituyentes.

Por último, nos interesa hacer notar la incapacidad del clítico para recibir una acentuación entonativa que lo destaque sobre el resto de los constituyentes de la oración:

(33) a. *Te LAS tomás ya Juan.

b. *Se LAS da de inteligente siempre.

En relación con esta última prueba nos parece importante indicar que A. Cardinaletti (2011) menciona el hecho de que los elementos que no pueden recibir acento, pueden ser palabras llenas (completas) pero es difícil que se comporten como sintagmas completos.

\section{CONCLUSIONES Y PROYECCIÓN PARA LA INVESTIGACIÓN}

Este trabajo presenta un problema que se puede situar entre la morfología y la sintaxis pero cuyas consecuencias tienen alcance sobre la constitución del lexicón. Las locuciones que hemos analizado no son nuevas en la variedad argentina, especialmente de la zona del Río de La Plata, pero se encuentran en uso y el proceso por el que se han formado presenta características interesantes. Se trata de un proceso que se sitúa en la interfaz entre la morfología y la sintaxis. Es importante notar que el grado de lexicalización alcanzado podría seguir o no. Es decir, creemos que no es posible que el verbo deje de flexionar en tiempo y modo porque justamente la característica de estas formaciones es que es el verbo el soporte más completo en cuanto a significado y morfología.

Podemos hipotetizar, teniendo en cuenta los rasgos morfológicos, sintácticos y semánticos considerados en este trabajo, que el punto de lexicalización hasta el que se ha llegado no se revertirá, en todo caso, lo que puede suceder es que las locuciones desaparezcan del uso.

Entendemos que la formación de compuestos o expresiones idiomáticas se hace por medio de los procedimientos lingüísticos comunes a todas las lenguas. El análisis 
que proponemos centra su atención en una clase de proceso de formación de locuciones de la variedad argentina del español, pero nuestro interés es comprender cómo se pueden crear conceptos a partir de la sintaxis. Una característica particular de las locuciones que hemos presentado es la presencia de un clítico vacío de contenido, como hemos mencionado antes, casi un expletivo. Es interesante notar que el español no dispone de expletivos en nominativo, a diferencia del inglés (it) o del francés (i), por lo que resulta sorprendente la capacidad de una variedad de la lengua para modificar el significado de un verbo a partir de la presencia de un clítico femenino plural en acusativo sin contenido semántico o, más acertadamente, sin capacidad referencial.

Interesa mencionar aquí dos tipos de procesos de formación de palabras que son muy comunes en la variedad que analizamos aquí que, si bien no están en nada relacionados con el que planteamos, son llamativos porque dan cuenta de la necesidad de generar neologismos estilísticos en el español de Argentina (especialmente en la variedad rioplatense). Se trata del truncamiento y del denominado vesre. El primero consiste en la caída del final de algunas palabras, sin que tal truncamiento tenga en cuenta, aparentemente, la morfología de las palabras en cuestión. Este procedimiento tiene un matiz despectivo que no valoramos aquí, solo lo mencionamos. Así, a partir de paraguayo se forma paragua, por ejemplo. El otro procedimiento también interesante, desde el punto de vista de la formación de neologismos, consiste en invertir las sílabas de una palabra y formar otra que conserva su significado pero que se usa en otros registros. Por ejemplo, se dice: ofri, jermu, jabru para referirse a frío, mujer, bruja. Este mecanismo de formación de palabras no tiene connotaciones despectivas y casi carece de otros matices, más que el de la creatividad en sí misma.

Dejamos planteada también la cuestión de seguir analizando los clíticos como elementos frasales o como morfemas. Entendemos que este es un problema que excede en mucho lo que hemos podido decir aquí, pero interesa seguir investigando sobre el tema. 


\section{BIBLIOGRAFÍA}

Academia Argentina de Letras (2008). Diccionario de Habla de los Argentinos. Buenos Aires: Espasa.

Aguirre, J. \& al. (2013). Puto el que lee: diccionario argentino de insultos, injurias e improperios. Buenos Aires: Gente Grossa.

Buenafuentes de la Mata, Cristina (2007). Procesos de gramaticalización y lexicalización en la formación de compuestos en español. Bellaterra: Tesis doctoral inédita, UAB.

Borer, H. (Ed.). (1986). Syntax and Semantics. Vol. 19: The syntax of pronominal clitics. Orlando, EEUU: Orlando Academic Press.

Cabré, M. ${ }^{a}$ T. (2008). La neología efímera, en M. ${ }^{a}$ T. Cabré, J. Freixa y E. Solé (Eds.), Lexic i neologia. Observatori de neologia. Barcelona, España: IULA, pp. 13-27.

Camacho Taboada, M. ${ }^{a}$ V. (1998). El concepto de clítico en la teoría gramatical. Interlingüística, 9, 81-84.

Cardinaletti, A. (2011). German and Italian modal particles and clause structure. The Linguistic Review, 28/4, pp. 493-531.

Delbecque, N. (2009). El femenino plural: marcador morfológico de unidades lexicalizadas, en R. De Maeseneer, I. Jongbloet, L. Vangerhuchten, A. V. Hecke y J. Vervaeke (Eds.), El hispanismo omnipresente, Bruselas: University Press Antwerp, pp. 145-167.

Ghio, A. \& Albano, H. (2015). 'Locuciones verbales' con pronombre personal átono la/las en el español coloquial de Buenos Aires, Argentina. Gramma, XXIV, pp. 102-116.

Jackendoff, R. (1990). Semantic Structures. Massachusetts: Cambridge University Press.

Lucena Cayuela, N. (Ed.) (2012). Diccionario de Uso del Español de América y España VOXUSO. Barcelona, España: SPES.

Mendívil Giró, J. L. (1999). Las palabras disgregadas. Sintaxis de las expresiones idiomáticas y de los predicados complejos. Zaragoza: PUZ.

Panagiotidis, P. (2002). Pronouns, clitics and empty nouns: pronominality and licensing in syntax. Amsterdam: John Benjamins Publishing.

Pustejovsky, J. (1995). The Generative Lexicon. Massachusestts: MIT Press.

Real Academia Española (2014). Diccionario de la Lengua Española. (R. A. Española, Ed.) Madrid: Espasa http://dle.rae.es. 